\title{
ON A STATIC AND DYNAMIC CALIBRATION OF THERMOCHROMIC LIQUID CRYSTALS
}

\author{
Stanislav Solnař*, Jan Medek, Abubakar Shola Suleiman, \\ PATRIK VYHLÍDAL \\ Czech Technical University in Prague, Faculty of Mechanical Engineering, Department of Process Engineering, \\ Technická 4, 16000 Prague 6, Czech Republic \\ * corresponding author: stanislav.solnar@fs.cvut.cz
}

\begin{abstract}
The paper deals with a static and dynamic calibration of selected wide-range thermochromic liquid crystals (TLC - SolarDust 24C), which are very easy to ingest and can provide accurate information on the temperature distribution on different surfaces. Static calibration problems, such as TLC illumination angle or illumination intensity, are solved and conclusions are drawn for the application of such a measurement. Dynamic experiments also indicate a certain time delay (around 50 ms) of the applied TLC measurement layer, which is very important to know for dynamic experimental methods, but the error and inaccuracy of the experiment is too high to draw conclusions.
\end{abstract}

KEYWORDS: Thermochromic liquid crystals, TLC, static, dynamic, calibration.

\section{INTRODUCTION}

Temperature measurement is a very important aspect of perhaps every industry, from heavy machinery to the very precise pharmaceutical industry. Temperature affects all mechanical, thermophysical and process parameters, so its knowledge is usually critical.

For many common applications of a (even very accurate) temperature measurement, we use touch (contact) thermometers, which are based on various physical principles (e.g., thermal expansion of substances or a thermal change of electrical resistance of conductors). In cases where it is not possible to measure the temperature by contact or the temperatures are very high, we can use contactless measurements using IR cameras. These cameras measure the intensity of the emitted infrared radiation or its wavelength and thus evaluate the surface temperature of the measured object. Such a measurement can also be very accurate, but IR cameras are usually very expensive and other phenomena, such as surface emissivity, reflections, etc., can affect the quality of measurements $([1], 2])$.

Thermochromic liquid crystals, which are both relatively inexpensive and easy to use, can offer very simple and accurate surface temperature measurements. We most often encounter TLCs as a liquid that is sprayed on the monitored surface or in the form of self-adhesive plates. At the same time, these thermochromic liquid crystals have often been used for scientific experiments in the past, so their reliability is considered to be very good.

From a physical point of view, it is a substance in the narrow range between the liquid and crystalline phases, in the literature, this state is also called mesophase. This mesophasic state allows the undissolved crystals to have different orientations in the fluid depending on the ambient conditions, e.g. UV radiation, electric field voltage or temperature. It is the temperature dependence of the inclination of the crystals that is very often used, because a change in the temperature of the TLC layer leads to a change in the visible colour of the surface. A change in the rotation of the individual crystals leads to a change in the refraction of the incident white light. The first mesophasic substance dates back to the end of the 18th century, and a large number of them have been described since then. From a chemical point of view, these are mostly crystallic organic molecules in the right temperature interface. Several mesophases of minerals are also already known $([1], 3])$.

TLC layers have been used successfully in various scientific fields, for example, Newton et al. 4] have used a thin layer of TLC to measure the convective heat transfer coefficient in gas turbine cooling ducts. In the experiments, the layer was applied to the monitored surface and the authors used the "slow transient" method to minimise errors in the step change. This method leads to the solution of a non-stationary onedimensional Fourier equation and it can be assumed that the delay of the measuring TLCs is so small that no fundamental measurement error occurs.

Ireland and Jones [5] have successfully applied similar measurements of the heat transfer coefficient and, in addition, have prepared measurements of surface tension on the wall in their article. The beginnings of similar experiments date back to the 80's and on the basis of pre-calibrated positions of the experiment, it is possible to evaluate the surface tension. The experimental surface is illuminated with white light at a given angle and then the camera captures the monitored surface from different angles. This surface is coated with TLCs and is affected by the air flow. Based on the evaluation of the surface temperature 
from different measurement angles, it is then possible to recalculate the surface tension value.

The article also mentions their earlier work on the dynamic behaviour of TLCs, however, the authors only state that the delay was measured in the order of milliseconds.

In addition, Stasiek et al. 6 have shown the use of TLCs in biomedicine, where we can meet with measurements that can non-invasively detect cancer. The TLC layer is applied to the skin surface and the temperature field in this area is monitored. Due to other thermophysical properties of the tumor, it is possible to evaluate whether the tumor is in this area or not.

Due to the width of the application of TLCs, we can encounter their application very often. However, a very important part of accurate TLC measurements is also their calibration, which is necessary for each applied layer. The calibration curve represents the dependence of the surface colour shade on the temperature, and in the literature, we can find a large number of articles that deal with the static calibration of the TLC layer.

One article that should be cited is Rao and Zang [7, who prepared an article on the application, calibration, and inaccuracy of wide-band TLC measurements. At the beginning of the article, they presented their experimental apparatus, which consisted of an isolated calibrator and an isothermal copper plate. They investigated whether the quality of the TLC coating affects the measurement and concluded that thicker TLC layers $(20-40 \mu \mathrm{m})$ lead to more accurate measurements and lower measurement inaccuracies. According to the authors, the quality of the TLC application on the surface has a very fundamental effect on the measurement and can halve the measurement uncertainty. They also described that the angle of inclination of the light has an effect on the absolute value of the measurement, however, the effect on the opacity is minimal. The authors also recommend using a median filter to reduce the impact of calibration on the digital noise generated during the measurement.

Their work is very extensive and we consider it a very good basis for their own TLC calibration measurements.

\section{Static CALiBration}

The measuring device for a static TLC calibration is based on a comparison method between a colour image from an RGB camera (Go Pro Hero 5 Black) with a fixed setting (ISO 400, WB 4000K and shutter speed $1 / 25 \mathrm{~s}$ ) and an accurate Pt 1000 thermometer (Greisinger GMH 175). The constant temperature in the calibrator was maintained by a water thermostat (Lauda E-100). A polarizing filter (Hoya CIR-PL) was installed in front of the RGB camera lens to prevent light from reflecting off the captured image. All components were placed on a solid sheet metal and placed in the dark to avoid any ambient optical influences. A sufficient light for a correct evaluation of the surface colour of the TLC layer is guaranteed by camera LED lights YN-14 40. These LED lights allow us to set the amount of lighting as well as the temperature of the light, which was set to $4000 \mathrm{~K}$ for all experiments. The camera was placed on the measuring axis at a distance of $300 \mathrm{~mm}$ from the measuring plate, the lights were set to an angle of 45 degrees at a distance of $200 \mathrm{~mm}$ from the measuring axis. The details of our static experiment are shown in Figure 1 .

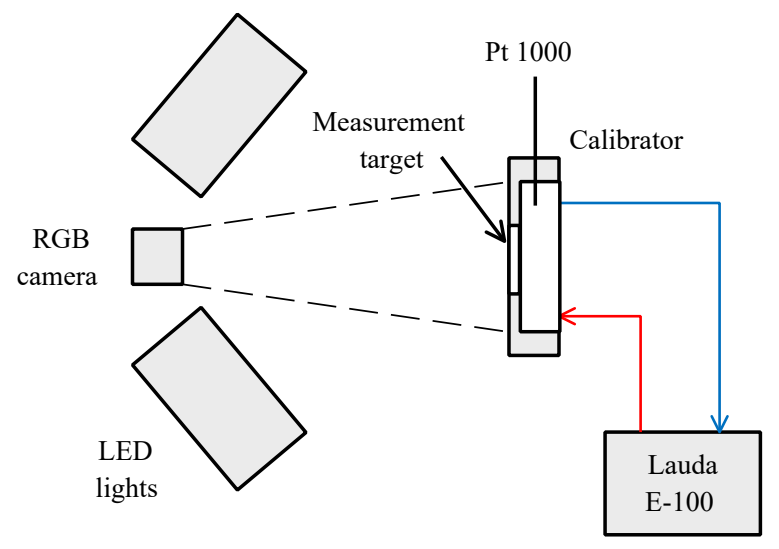

FIGURE 1. Schematic drawing of a static calibration experiment. RGB camera (Go Pro Hero 5), calibrator, thermometer Pt 1000 and LED lights (YN-14 40) are firmly placed on a solid metal sheet. During the experiment, the entire assembly is covered with a wall and a black cloth to minimise the ambient light and optical phenomena.

The calibrator was made of plexiglass, which has a very poor thermal conductivity, and thus, losses to the environment were minimised. The measuring plate itself was made of steel (thickness $1 \mathrm{~mm}$ ) and a layer of measured TLCs was provided on the outside. The design of the calibrator minimised the possibility of natural convection around the calibrator. A schematic drawing of the static experiment calibrator is in Figure 2

Prior to the application of the wide-band TLC layer (SolarDust 24C, range $60-90 \mathrm{~F}$, approx. $16-32$ ${ }^{\circ} \mathrm{C}$ ), a thin layer of matt black paint was applied to the measured metal target, which is recommended for an easier reading of the colours of the TLC layer. A total of 7 layers (about $2.5 \mathrm{ml}$ before drying) of TLCs were then applied to the black coating using an air brush for a perfect layer distribution and thickness minimization. The layers were applied in a perpendicular arrangement to the previous layer. This created a very high-quality painted surface, which did not show any defects. Due to the controlled conditions of the measurement environment, we did not apply any covering transparent layers to the TLCs.

Before each measurement, the temperature in the calibrator was allowed to settle for 10 minutes before the surface colour image of the TLC layer was taken 


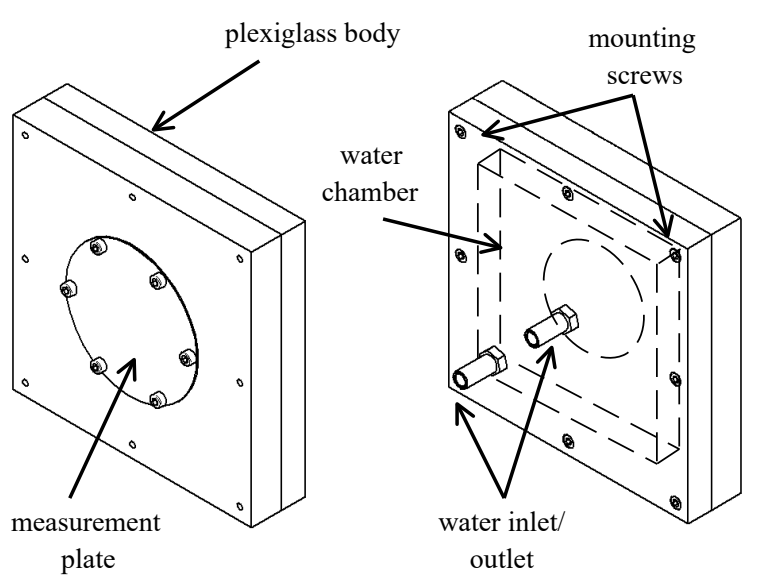

Figure 2. Drawing of a static calibrator made of plexiglass with a metal measuring plate. The external dimensions of the calibrator are $200 \times 200 \times 40 \mathrm{~mm}$, the measuring plate is a circular plate with a diameter of $110 \mathrm{~mm}$ and a thickness of $1 \mathrm{~mm}$. Inside the calibrator is a water chamber with a size of $140 \times 140 \mathrm{~mm}$, which is milled in the area of the measuring plate up to the measuring plate.

and the temperature was read from Pt1000. From the RGB camera, we obtain an image (or several images), of which each point consists of three layers (R, G and B). However, the RGB model of the colour representation is not well suited for this experiment, because it takes into account other shades of the same colour. The HSV (or sometimes HSB) model is commonly used in the literature for the evaluation, because the Hue value (degree of colour) is not affected by the colour shade or its lightness. There are several equations for the conversion of the RGB and HSV models, we used the rgb2hsv command in the MATLAB environment, which is based on the dominant colour. If the red channel is dominant, then

$$
\text { Hue }=\frac{G-B}{6(R-\min (R, G, B))},
$$

if the green channel is dominant, then

$$
\text { Hue }=\frac{2+B-R}{6(G-\min (R, G, B))}
$$

and if the blue channel is dominant, then

$$
\text { Hue }=\frac{4+R-G}{6(B-\min (R, G, B))} .
$$

After this transformation, we obtain a matrix of Hue values that is not affected by the brightness or shadowness of the colour in any way.

To eliminate the noise that occurs when taking digital photos, we scanned the entire measured target and evaluated a selected area of points in the middle of the target (usually $500 \times 500$ measured pixels). A typical dependence of the Hue value on the temperature (also known as a calibration curve) for our type of the TLC layer can be seen in Figure 3 .

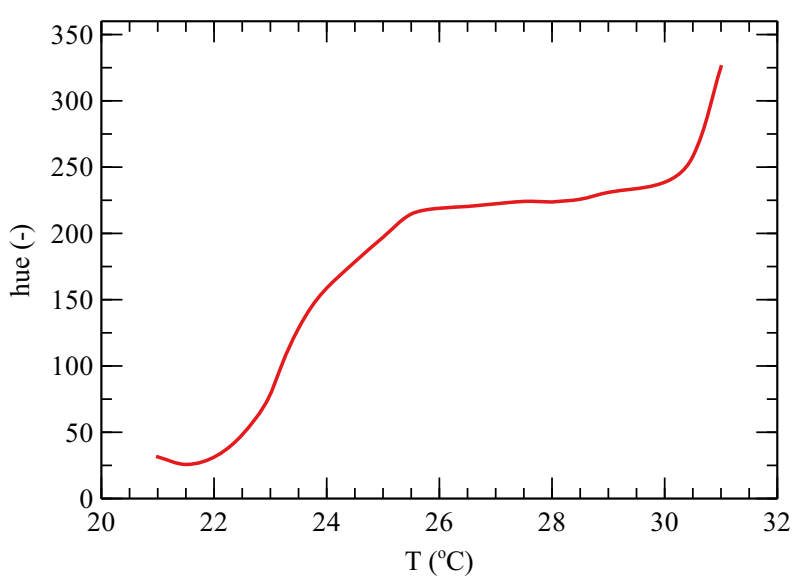

Figure 3. Typical temperature dependence of the Hue value for our type of the TLC layer. This is a very nonlinear dependence, which does not even have a clear tendency at the ends. In practice, a narrower strip is usually used, where the tendency is unambiguous and the sensitivity of the layer to temperature changes is high.

The calibration curve also shows that the TLC layer has various sensitivities to temperature changes. In the region around $24^{\circ} \mathrm{C}$, the sensitivity is about 85 hue $/{ }^{\circ} \mathrm{C}$, while in the region around $28^{\circ} \mathrm{C}$, the sensitivity is only about 3 hue $/{ }^{\circ} \mathrm{C}$.

For TLC measurements, it is also very important to find out if there is only one colour in the image or if the evaluated image is composed of many different Hue values (colours). For this confirmation, we prepared a histogram of the colour distribution in the evaluated field of the image, see Figure 4

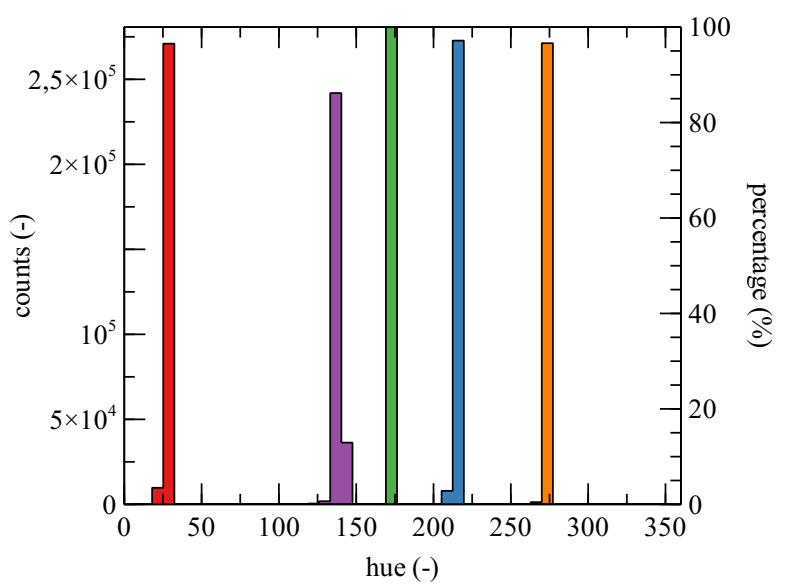

Figure 4. Histogram of the distribution of individual Hue values for 5 various cases. It is obvious that only one Hue value is always dominant. One image usually contains more than $98 \%$ Hue of one colour (98\% of measured pixels show one colour).

A measurement using this TLC layer is, therefore, possible, one colour is always dominant in the measurement and so the evaluation can be carrioud out without any problems. The temperature dependence 
of the Hue value (calibration curve) is strongly nonlinear and has various sensitivities to temperature changes.

\subsection{Influence of the Amount of Light}

For the same measuring target with the TLC layer applied, we verified whether the amount of light affects the result of the measured values. The LED lights used allow to adjust the amount of light in several steps, for the experiment, the settings of 25, 50, 75 and $100 \%$ intensity were used. The experiment was basically the same as the static calibration (comparison method) with different lighting conditions and the results are shown in Figure 5

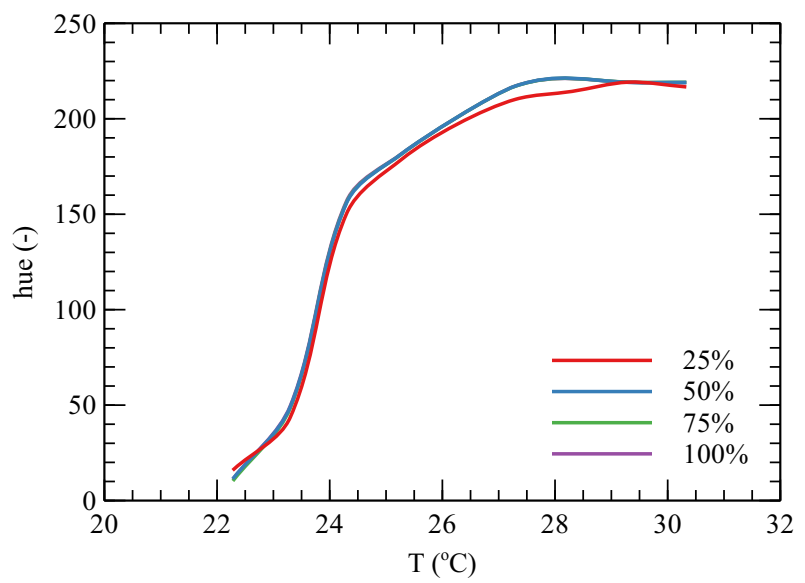

FiguRE 5. Comparison of calibration curves for different light intensities.

Although the calibration curves do not show a significant difference, it is clear that a deviation is noticeable for a lighting level of $25 \%$. If we consider a calibration curve with an illumination level of $100 \%$ as a reference, we can plot the deviations of other calibration curves and find out the deviations, see Figure 6.

Based on our measurements, we can judge that if we use an illumination intensity greater than $25 \%$, the deviation from the reference calibration curve (illumination intensity $100 \%$ ) will be minimal. We detected a larger deviation at the beginning of the calibration curve, but if the measurement will take place in the range from $24{ }^{\circ} \mathrm{C}$ upwards (the area of the highest sensitivity of the TLC layer to temperature changes), this deviation is also negligible. We do not recommend measuring with a very limited lighting intensity

\subsection{INFLUENCE OF THE ANGLE OF LIGHT}

We also investigated the effect of light tilt on the measured values by the same method as the calibration curve and the effect of light intensity. We have adjusted the experimental setup for the possibility of moving the lights. The relocated lights then always aimed at the center of the measuring plate.

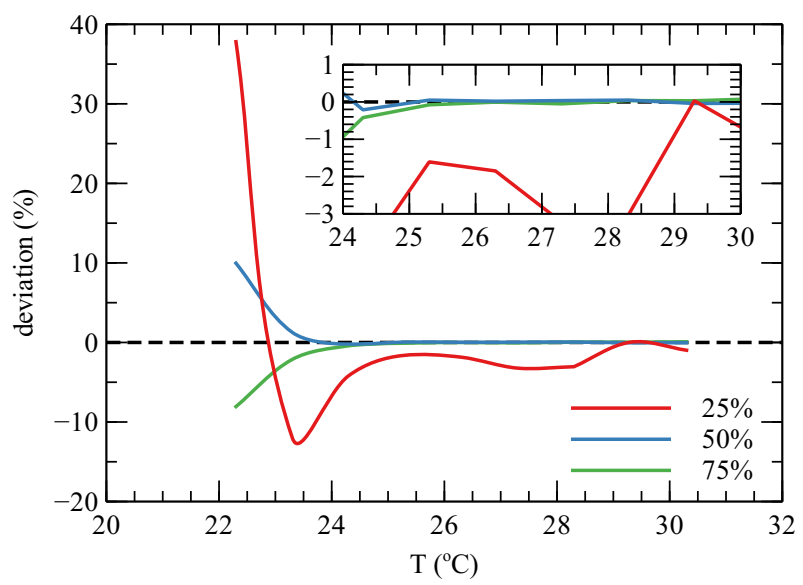

Figure 6. Deviations from the reference calibration curve. We can see fundamental deviations at the beginning of the calibration curves and also for the whole curve with an illumination level of $25 \%$. Other curves have a deviation of less than $1 \%$ from the reference.

Three light setting angles (36, 45 and $66^{\circ}$ ) were selected for the measurement to observe changes in the measurement. The changes in the calibration curves are clearly visible in this experiment, see Figure 7

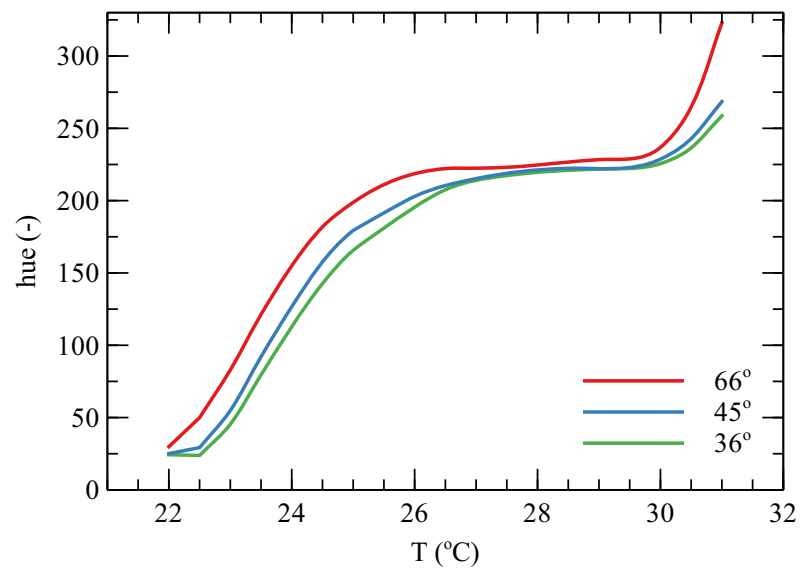

FiguRE 7. Comparison of calibration curves for different light angle settings.

From the comparison of the calibration curves, we can conclude that with an increasing angle of light, the value of Hue also increases, and the measurement must, therefore, be approached with a uniform setting of the angle of light. For a comparison, we again chose one reference calibration curve (in this case with an angle of $45^{\circ}$ ) and monitored the deviations of the other curves, see Figure 8

Although all calibration curves have a very similar trend and show a similar sensitivity in individual sections of the curve, the differences are not negligible and the shapes are partially different. Nor does there appear to be a difference that can be expressed by any simple multiple constant. From this point of view, 


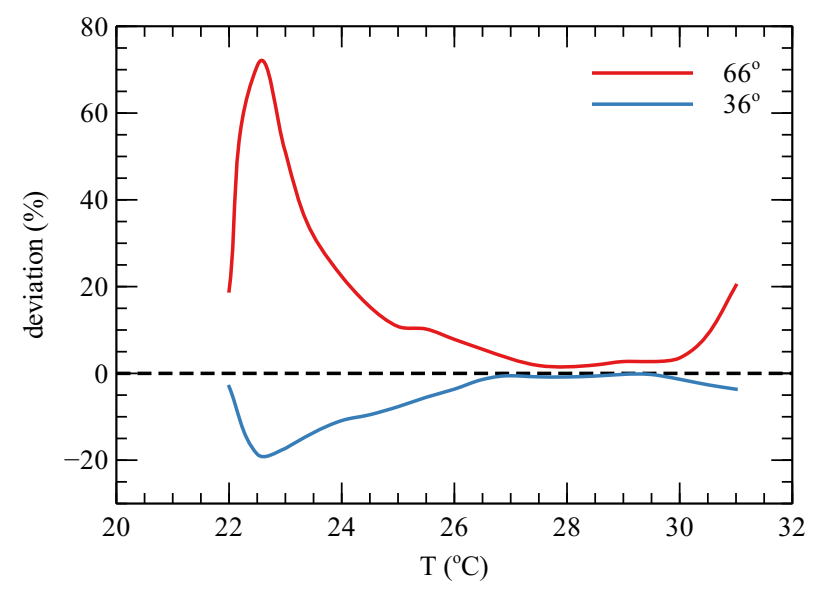

FiguRE 8. Deviations of the calibration curves from the reference curve. We can see deviations in the tens of percent, which approach below $10 \%$ only in the range of $26-30^{\circ} \mathrm{C}$.

it is critical to know the illumination angle and to prepare a calibration curve for this setting before the actual measurement.

\section{DyNAMIC CALIBRATION}

The static calibration has shown that with some limitations (especially knowledge of the angle of incident light), TLCs can be used for static experiments. For dynamic experiments (eg thermal oscillation method, step or response methods), however, it is also necessary to know the dynamic properties of the TLC layer - its delay compared to the excitation function or other time deviations.

To monitor the dynamic behaviour of the applied TLC layer, we prepared a similar experiment, which consisted of two measuring bars, which were connected together by a delay channel. The calibrator for the dynamic behaviour was made by $3 \mathrm{D}$ printing (mat. ABS - very bad thermal conductivity $k=0.25 \mathrm{~W} /(\mathrm{mK})$, Prusa i3 MK3) and sealed by etching the walls with acetone vapour. A model of the calibrator can be seen in Figure 9

The 3D printed body has two windows on which a TLC layer is applied. In this case, a PMMA plate was used as the measuring plate, and a TLC layer was applied to the inside of the calibrator in the same number of layers and the same pattern. The TLC layer thus prepared was then covered with a spray layer of black matte paint for a correct colour identification. The black colour also served as a barrier to washing away the TLC layer. The measuring targets thus prepared were then fastened to the calibrator body with screws and a delay loop was fitted.

For observation possibilities, the whole calibrator was also monitored by an IR camera (MicroEpsilon TIM $160,160 \times 120$ pixels, $80 \mathrm{mK}$ sensitivity). The emissivity of the PMMA surface was measured by a comparative static method and was determined to

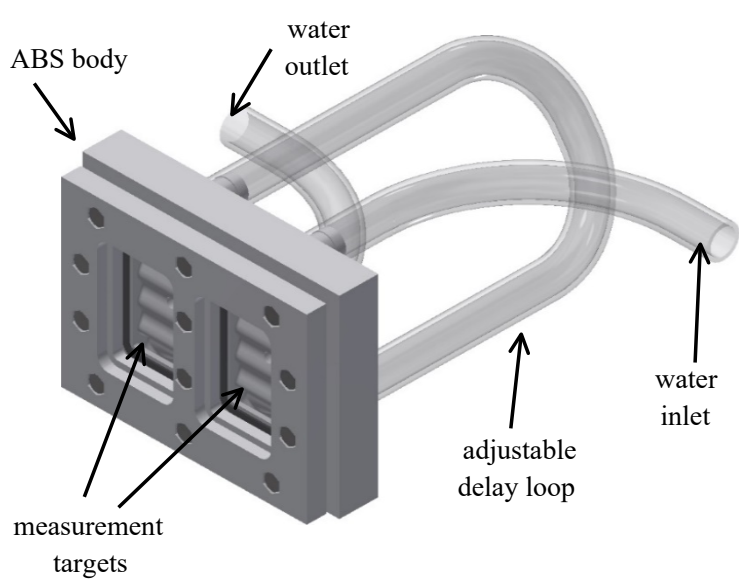

Figure 9. Model of our dynamic calibrator with an adjustable delay loop. During the experiment, all tubes are provided with thermal insulation to minimise the losses to the environment, see $[3$.

be $\epsilon=0.73$. Although the TLC layer is deposited on the inside and the IR camera senses the surface temperature, we assume that both signals are comparable. Since both measuring targets are made of the same material of the same thickness, we assume that the heat will be conducted in them at the same speed and due to the insulation of the calibrator, to approximately the same temperature. This assumption leads to the possibility of comparing the measured colour shade by the TLCs (time dependence) and the time dependence measured by an IR camera. Another possibility is the use of very fast thermocouples, which will be close to the measured target from the inside, which is the goal of our further research.

To measure the dynamic behaviour, we prepared an experiment with two cameras, see Figure 10. With both cameras, we recorded temperature jump changes, which we performed with hot and cold water (stored in tanks), which was alternated manually in a 3-way valve. Due to the thermal capacity of the system, however, there are no step changes, but continuous gradual changes in both spectra (both visible and IR).

It is possible to use several methods to evaluate continuous changes in signals, we used the method of finding the inflection point that we think best describes the change. Both cameras were set to a high frame rate $(120 \mathrm{~Hz}$ for the RGB camera and $100 \mathrm{~Hz}$ for the IR camera) and captured the experiment. Theoretically, the time resolution of this experiment is $10 \mathrm{~ms}$ of time response. The synchronization of signals from the IR and RGB cameras was performed manually using an optical element.

From both measured windows, the field in the middle of the measurement was selected, which had dimensions of about $10 \times 10 \mathrm{~mm}$ and the corresponding camera resolution (IR and RGB camera have different resolutions). Scanning a part of the measured area also helped to eliminate any digital noise. The scanned field was always averaged to the final value 


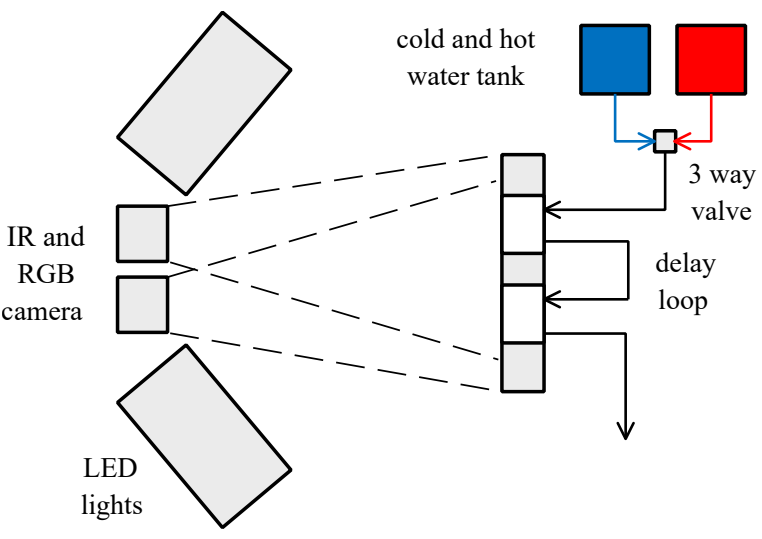

Figure 10. Schematic drawing of an experiment for measuring the dynamic behaviour of a TLC layer. The distance of the cameras from the measuring windows is about $300 \mathrm{~mm}$.

(colours or temperatures).

The measured temperature profiles (both from the TLCs and from the IR camera) show a very similar course (continuous changes), both during the heating and the cooling. By evaluating the course of heating and cooling, we obtain the same time difference (with an error of about $\pm 10 \mathrm{~ms}$ ) for both the TLC and the IR camera. The detection of the system is, therefore, efficient and both temperature changes (heating and cooling) can be used for a system time delay evaluation.

The evaluation of time dependences (a comparison of time delays of the TLC and IR) shows that the difference between the two measured signals is in the order of tens of milliseconds, the average value is about $49 \mathrm{~ms}$.

But we must take into account the inaccuracy of this experiment. If we assume that the scanning error of both IR and RGB cameras is a maximum of \pm 1 frame, we obtain a systematic error of $\pm 10 \mathrm{~ms}$ of measured signal differences. We create another introduced error by fitting the function to the measured data and searching for their inflection point. If we again assume that this operation creates an inaccuracy of only \pm $10 \mathrm{~ms}$ and we also consider other inaccuracies (digital noise, random errors, etc.), we find that the measured value of the TLC signal delay is about as large as the inaccuracy of the experiment.

Therefore, we do not recommend the use of this experimental device to determine the dynamic behaviour of TLCs. Either the TLC delay would have to be in the hundreds of milliseconds, or more (maybe only faster) sophisticated experimental equipment must be used. However, this experiment showed that TLC delays can be expected in the order of tens of milliseconds.

\section{Discussion}

The article discusses the issue of calibrating thermochromic liquid crystals (TLC), which very often serve as temperature indicators. The great advantage of TLCs is the very low cost and ease of application when measuring. The disadvantage is their sensitivity to damage and usually a narrow range of measured temperatures.

For the experiments, we used a wide-range TLCs labeled SolarDust $24 \mathrm{C}$, which has a range of approximately $16-32{ }^{\circ} \mathrm{C}$. However, for each experimental measurement, it is necessary to calibrate any measuring element. While carrying out the experiments, we also discovered several changes that interested us and we tried to analyse them.

The calibration curve (surface temperature dependence and TLC colour shade) is a very non-linear curve that changes the sensitivity from 5 hue $/{ }^{\circ} \mathrm{C}$ to 85 hue $/{ }^{\circ} \mathrm{C}$. From the point of view of experimental use, it is most advantageous to use a narrower band of measured values, where the sensitivity to the temperature change is the highest.

The data show that only one colour shade is always dominant for the set temperature, which is crucial for the application. The measured data contained $98 \%$ or more pixels of the same shade of colour.

We found that the light intensity has no significant effect on the change in the calibration curve. Only when the lighting is insufficient, there is a fundamental deviation and this is also accompanied by a greater digital noise. When we used a sufficient high-power illumination in the experiments, we recorded a deviation from the reference calibration curve of about $1 \%$ and about $8 \%$ at the beginning of the measurement interval.

The data show a fundamental influence of the calibration curve in terms of the angle of adjustment of the lights and this is essential for experimental measurements. For a further use of TLCs, we therefore recommend using the same light angle setting, or obtaining a calibration curve for the current experiment settings. Deviations from the reference calibration curve were significant in the order of tens of percent.

Dynamic experiments have shown that there is probably some time lag of the TLC layer to the temperature change. The data show that this delay can be expected in the order of tens of milliseconds, but the inaccuracy and insensitivity of the experiment (in the order of tens of milliseconds) does not allow conclusions to be drawn. For a more detailed examination of the dynamic behaviour of TLCs, we therefore recommend using the same experiment, but with a higher sensitivity, or using another dynamic experimental technique that would be able to detect these dynamic phenomena with a reasonable error.

For further experimental measurements, we recommend shading other ambient light effects using a box or black fabric curtains. We also recommend using a large amount of heat transfer medium (in our case water) to obtain the largest possible heat capacity of the measuring system and thus the smallest possible temperature fluctuations in time. In our experiments, professional camera lights with their own voltage sta- 
bilization proved to be very successful, other lights did not guarantee a uniform light colour.

\section{Conclusion}

From our experimental measurements we can conclude:

- calibration curve of chosen TLC layer is very nonlinear with varying sensitivity to temperature change

- experimental measurements with our camera always show only one dominant colour in the whole image, so it is possible to use TLCs for surface temperature measurements

- light intensity has no significant effect on the change in the calibration curve

- changing the illumination angle has a significant effect on the calibration curve

- dynamic experiment prepared by us shows a time delay of about $50 \mathrm{~ms}$, but it is necessary to create a more accurate measurement to detect the dynamic behaviour

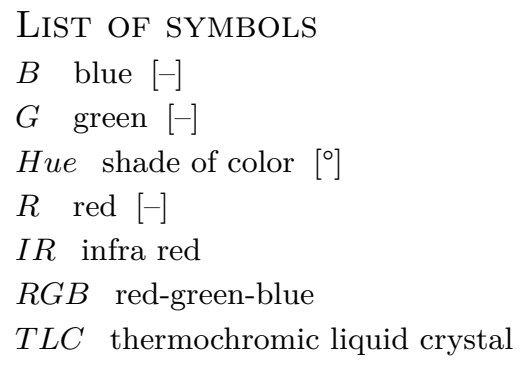

\section{ACKNOWLEDGEMENTS}

The main author would like to thank the other colleagues for their excellent cooperation during their thesis. Au- thors acknowledge the support from the Grant Agency of the Czech Technical University in Prague, grant no. SGS20/119/OHK2/2T/12.

\section{REFERENCES}

[1] P. Vyhlídal. Problems of surface temperature measurement using themochromic crystals (in Czech). Master's thesis, Czech Technical University in Prague, Faculty of Mechanical Engineering, 2019.

[2] A. S. Suleiman. Thermochromic liquid crystals as a temperature indicator. Master's thesis, Czech Technical University in Prague, Faculty of Mechanical Engineering, 2019.

[3] J. Medek. Thermochromic liquid crystals (TLC) calibration (in Czech). Master's thesis, Czech Technical University in Prague, Faculty of Mechanical Engineering, 2020.

[4] P. J. Newton, Y. Yan, N. E. Stevens, et al. Transient heat transfer measurements using thermochromic liquid crystal. part 1: An improved technique. International Journal of Heat and Fluid Flow 24(1):14-22, 2003. https://doi.org/10.1016/S0142-727X (02)00206-0

[5] P. T. Ireland, T. V. Jones. Liquid crystal measurements of heat transfer and surface shear stress. Measurement Science and Technology 11(7):969-986, 2000. https://doi.org/10.1088/0957-0233/11/7/313

[6] J. Stasiek, M. Jewartowski, T. A. Kowalewski. The use of liquid crystal thermography in selected technical and medical applications - recent development. Journal of Crystallization Process and Technology 4(1):46-59, 2014. https://doi.org/10.4236/jcpt.2014.41007

[7] Y. Rao, S. Zang. Calibrations and the measurement uncertainty of wide-band liquid crystal thermography. Measurement Science and Technology 21(1):1-8, 2010. https://doi.org/10.1088/0957-0233/21/1/015105 


\section{A. Attachments}

MATLAB script for the evaluation of measured data. The measured data are saved in .jpg format and the file name is identical to the measured temperature multiplied by 10 (for easier data storage, there are no decimal points).

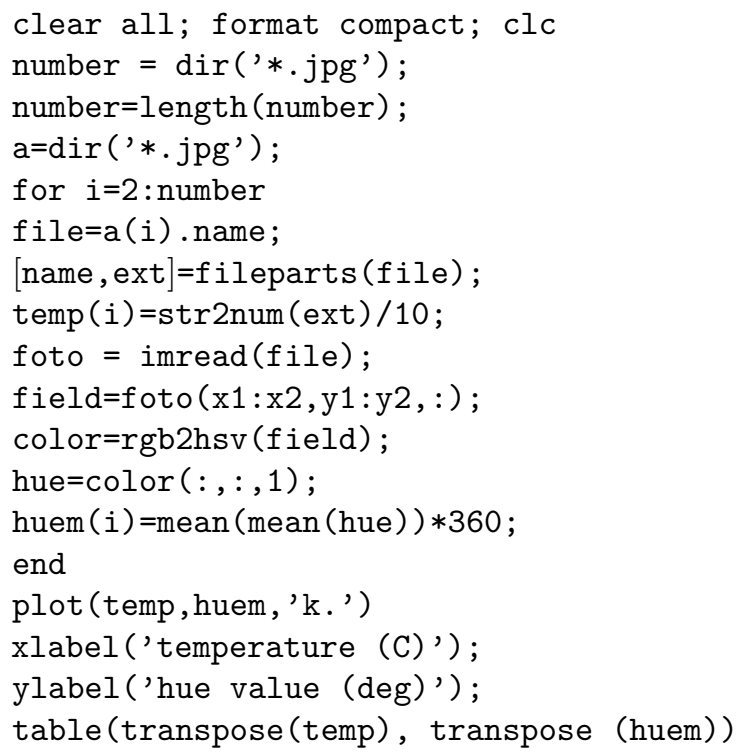

